\title{
Alcohol abuse and acute lung injury and acute respiratory distress syndrome
}

\section{Introduction}

Alcohol is one of the most commonly used and abused beverage worldwide. Alcohol is known to have numerous systemic health effects, including on the liver and central nervous system. From a respiratory standpoint, alcohol abuse has long been associated with an increased risk of pneumonia. More recently, alcohol abuse has been strongly linked in epidemiologic studies to development of ARDS in at-risk patients. The first demonstration of an association between chronic alcohol abuse and ARDS was made by Moss et al, who retrospectively examined 351 patients at risk for ARDS. ${ }^{1}$ In this cohort, $43 \%$ of patients who chronically abused alcohol developed ARDS compared to only $22 \%$ of those who did not abuse alcohol, with the effect most pronounced in patients with sepsis. This study was limited by its retrospective design, particularly since this design required that alcohol use history be obtained by chart review and documented history; furthermore, this study did not adjust for concomitant cigarette smoking. Encouraged by these findings, Moss et al conducted a multicenter prospective study of 220 patients with septic shock to further assess this relationship. Methodologically, this study improved on its predecessor by using the Short Michigan Alcohol Screening Test (SMAST), which has previously been validated as a screening test for chronic alcohol abuse. A multivariate analysis again found that those who chronically abused alcohol developed ARDS more frequently than those who did not, $70 \%$ vs. $31 \%$, respectively. These two key studies thus served as the first major evidence that alcohol use was a risk factor for the development of ARDS.

Several studies have since reinforced the relationship between alcohol use and ARDS. Licker et al examined the incidence of ARDS in 879 non-small cell lung cancer patients undergoing thoracic surgery. Multivariate logistic regression found that preoperative chronic alcohol consumption was associated with increased odds of developing acute lung injury. In addition, two studies examining risk factors for transfusion-related acute lung injury (TRALI) found that chronic alcohol consumption was associated with the development of TRALI. Gajic et al found that patients who developed TRALI were more likely to be chronic alcohol users when compared to matched controls, $36.5 \%$ vs. $17.6 \%$ respectively. More recently, Toy et al found that in a multivariate model, chronic alcohol use in patients receiving blood product transfusions significantly increased the odds of developing TRALI. A later study by Gajic et al that evaluated 5584 patients at risk for ARDS to determine a lung injury prediction score found alcohol to be a positive risk factor for the development of ARDS. These studies thus supported the prior observations and solidified the association between chronic alcohol use and ARDS.

Numerous studies have been performed both in animal models and humans to better understand the association between chronic alcohol use and ARDS. These studies have identified a central role for pulmonary immune dysfunction as well as alveolar epithelial dysfunction in the mechanistic link between alcohol and ARDS. Pulmonary immune dysfunction: Both acute and chronic alcohol use can contribute to a dysfunctional pulmonary immune response. Acute alcohol use impairs neutrophil chemotaxis and function with
Volume 10 Issue 6 - 2018

\author{
Fadhil Kadhum Zwer Aliqa \\ Private clinic practice, Iraq
}

\begin{abstract}
Correspondence: Fadhil Kadhum Zwer Aliqaby, Private clinic practice, Iraq, Email drfadhel2@gmail.com
\end{abstract}

Received: December II, 2017 | Published: November 28, 2018

subsequent decreased phagocytosis and bacterial killing. Chronic alcohol use is similarly associated with altered neutrophil function and decreased superoxide production. Interestingly, chronic alcohol use decreases levels of granulocyte/macrophage colony stimulating factor (GM-CSF) receptor and signaling in lung epithelium, which has been shown to result in defective alveolar macrophage maturation. The net effect of these abnormalities is an increased pulmonary bacterial burden.

In addition to its effects on neutrophils, alcohol use has a variety of effects on cytokine production in the lung. While acute alcohol use has been shown to impair production of proinflammatory cytokines such as TNF- $\alpha$ and IL-1 $\beta$, which may predispose patients to pneumonia, chronic alcohol use has associated with increased levels of proinflammatory cytokines, such as TNF- $\alpha$, IL-1 $\beta$, and IL- 6 , in both human and animal studies. Recently, it is found that elevated levels of CCL-5 (also known as RANTES), which is a chemoattractant for a variety of immune cells, in the BAL fluid of chronic alcoholics. ${ }^{2}$ This increase in inflammatory cytokines appears to have a significant effect on downstream inflammation, as IL-6 was recently shown to play a key role in the pulmonary inflammatory response of alcoholic mice in a burn injury model. This altered cytokine profile in conjunction with decreased neutrophil and alveolar macrophage function is thought to contribute to the development of ARDS in alcohol abusers.

Alveolar epithelial dysfunction: In addition to its effects on the lung inflammatory response, chronic alcohol use may also predispose to ARDS development by causing increased pulmonary oxidative stress and alveolar epithelial dysfunction. These effects are mediated in part via the renin-angiotensin system (Figure 1). Chronic alcohol use has long been known to increase activation of this system, resulting in elevated levels of angiotensin II in humans. Angiotensin II may contribute to alveolar epithelial dysfunction through a variety of mechanisms, including via systemic effects on vascular tone and fluid retention as well as via localized effects such as promoting apoptosis of alveolar epithelial cells. In addition, angiotensin II activates NADPH oxidase in the lung, resulting in elevated levels of reactive oxygen species. This increase in reactive oxygen species results in depletion in alveolar levels of the key antioxidant glutathione (GSH) and increases in alveolar oxidized glutathione (GSSG), a phenomenon seen both in animal models and humans who abuse alcohol. Interestingly, patients with ARDS have been shown to demonstrate the same derangement with regards to pulmonary glutathione. This alteration in glutathione results in decreased antioxidant capacity in the lung and has further been linked to decreased surfactant synthesis and increased type II 
cell apoptosis. Additionally, the depletion of glutathione appears to increase levels of latent TGF- $\beta$, which subsequently contributes to baseline alveolar epithelial dysfunction, manifested by increased permeability and lung edema. The net result of increased activation of this pathway is an alveolar epithelium that is already dysfunctional and thus primed for developing ARDS when faced with an acute insult.

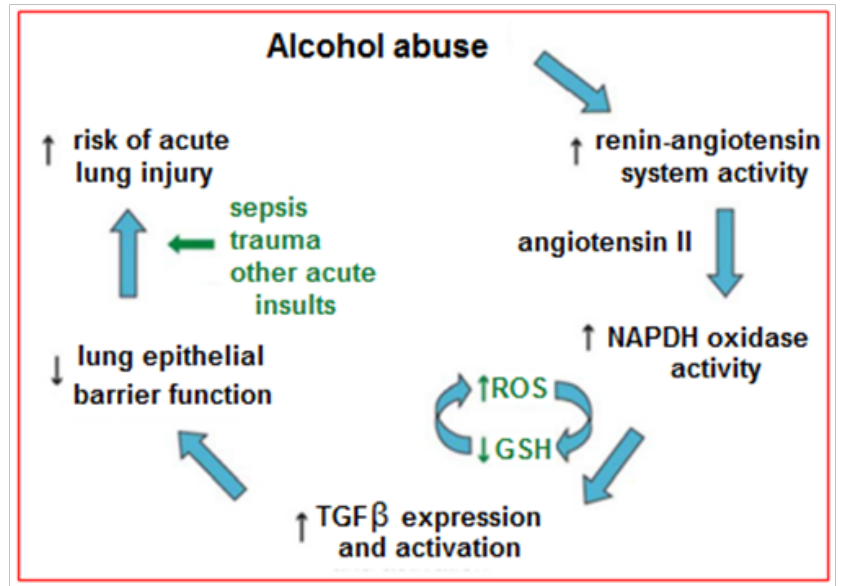

Figure 1 Potential mechanism by which alcohol primes the lung for Acute Respiratory Distress Syndrome.

Blood alcohol content, injury severity and acute respiratory distress syndrome: Blood alcohol content (BAC) $>0 \mathrm{mg} / \mathrm{dl}$ was present in $28 \%$ of the patients; most of those had a BAC $>100 \mathrm{mg} / \mathrm{dl}$. It was found that $\mathrm{BAC}>0 \mathrm{mg} / \mathrm{dl}$ was associated with an increased risk for ARDS development, this risk was dose related. Injury severity score (ISS) and Glasgow coma score (GCS) result from the injury event and occur after alcohol ingestion, making them intermediate outcomes rather than baseline characteristics. BAC $>0 \mathrm{mg} / \mathrm{dl}$ was associated with high ISS and low GCS. ISS and GCS were stronger predictors of ARDS development than BAC. Controlled experimental and epidemiological studies have shown alcohol exposure can increase the severity of injury. Epidemiologic studies that adjusted for injury severity in their analyses of outcomes from acute alcohol exposure may have obscured the association of mortality and other outcomes with BAC by over adjustment. It was showed an increased risk for ARDS development from $\mathrm{BAC}>0 \mathrm{mg} / \mathrm{dl}$ after adjusting for characteristics that were present prior to alcohol ingestion. Experimental studies with investigator control of injury severity are needed to better define the roles of acute alcohol exposure and trauma in the etiology of ARDS (Figure 2). Generally, elevated BAC is associated with ARDS development. $\mathrm{BAC}>0 \mathrm{mg} / \mathrm{dl}$ is associated also with severe injury (high ISS) and depressed sensorium (low GCS). Patients admitted with high ISS and low GCS are more likely than not to experience ARDS or die. In unadjusted analysis of the intermediate outcomes ISS and GCS, BAC $>0 \mathrm{mg} / \mathrm{dl}$ was associated with increased risk for high ISS $(\geq 16)$ and for low GCS $(\leq 8)$ (Figure 2). In adjusted analysis, BAC $>0 \mathrm{mg} /$ $\mathrm{dl}$ was associated with an OR 1.17 for high ISS and OR 2.52 for low GCS. Elevated BAC may increase the frequency of ARDS through influence on injury severity or independent molecular mechanisms which can be discriminated only in experimental models. High ISS and low GCS scores may be useful to identify patients at high risk for ARDS development. ${ }^{3}$

Effect of alcohol abuse on ARDS and multiple organ dysfunctions: In most hospitals about $21 \%$ of all ICU admissions are directly related to diagnosis resulting from alcohol abuse such as alcohol withdrawal syndrome and delirium tremens. Critically ill patients with a significant history of alcohol abuse are also admitted to the ICU for disorders not routinely appreciated to be modified by a prior exposure to alcohol. Because these patients cannot always communicate due to endotracheal tube and sedative agents, a history of alcohol abuse is not often obtained from them [but may be from some of their relatives]. Subsequently, important prognostic information may go unnoticed and specific alcohol-related interventions may not be routinely initiated. It was found that a history of chronic alcohol abuse was associated with a doubling of the mortality rate in nearly all patients that require ICU care. One of the first associations between alcohol ingestion and ARDS was reported in trauma patients. In the large cohort of trauma patients, those with a history of alcohol abuse had a higher incidence of respiratory complications. It was found that about $26 \%$ of the patients admitted to ICU developed ARDS with highest incidence in patients with sepsis syndrome (43\%) and those with multiple transfusions $(40 \%)$. Secondary risk factors that were also associated with the development of ARDS included an elevated APACHEII score greater than 20. A clear association between alcohol abuse and the development of ARDS was not clearly observed, however, the incidence of ARDS was about $70 \%$ in patients with a prior history of alcohol-related illness compared to $39 \%$ in those with no history of alcohol-related illness.

Association between Exposure, Intermediate Outcomes, and Primary Outcome

A.

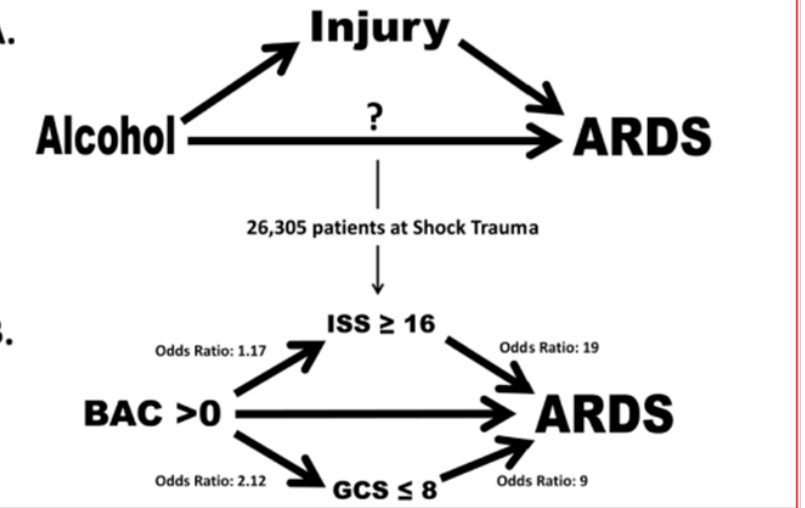

Figure 2 (A) Primary exposure of alcohol is temporally associated and occurs prior to injury which is an intermediate outcome prior to ARDS development. Alcohol may have a direct relationship with ARDS development also. (B) Unadjusted analyses from Shock Trauma Registry showing association between the primary exposure blood alcohol content with the intermediate outcomes ISS and GCS. The question of direct association of BAC with ARDS development cannot be answered without the ability to control the risk factors independently of each other.

Among the patients in ICU was also found in some studies that the incidence of ARDS was $70 \%$ in patients with a positive history for chronic alcohol abuse compared to about $31 \%$ in those without (Figure 3). This effect remained significant in a multivariate logistic regression model. The severity of organ dysfunction was determined using daily [sequential organ failure assessment] SOFA scores. Patients with a history of alcohol abuse had higher SOFA scores with about $60 \%$ with a score greater than 9 compared to $40 \%$ in those without an alcohol use history (Table 1). After adjusting for other confounding factors such as gender, age and source of infection, the severity of 
organ dysfunction in patients with a history of chronic alcohol abuse remained significant. Among the ARDS survivors, $40 \%$ had a history of alcohol abuse and hospital length of stay was significantly longer for these patients when compared to ARDS survivors without a significant alcohol history.

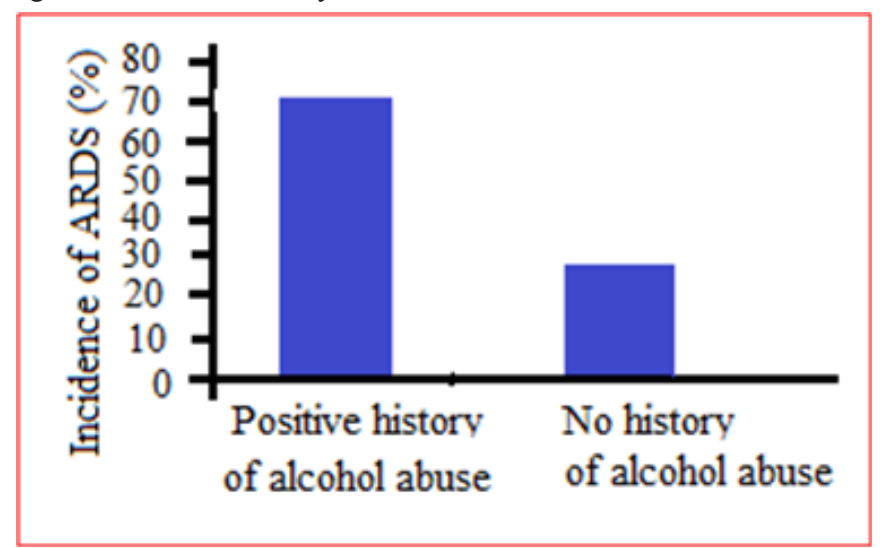

Figure 3 The incidence of acute respiratory distress syndrome when stratified by history of alcohol abuse

There are likely many potential mechanisms by which alcohol abuse increased susceptibility to developing ARDS (Figure 4). However, based on the extensive evidence implicating the role of glutathione deficiency in pathogenesis of alcohol mediated liver disease, it was postulated that glutathione may play a role in alcohol induced pulmonary dysfunction [vide infra]. Glutathione is an antioxidant that is involved in inflammatory responses and immune modulation, including the modulation of redox-regulated signal transduction, regulation of cell proliferation and remodeling of the extracellular matrix. Glutathione has also been shown to protect the airspace epithelium from free radical-mediated injury and inflammation. This tripeptide is produced in the liver. Alveolar epithelial type II cells import intact glutathione from the plasma and maintain a certain glutathione level in the epithelial lining fluid. In animal model of ethanol-mediated susceptibility to acute lung injury, it was found that ethanol feeding increased endotoxin-mediated acute edematous injury in lungs ex vivo and increased sepsis-mediated lung dysfunction in vivo. Compared to control-fed animals there was a decreased level of glutathione in the lung tissue, lavage fluid and inside alveolar type II cells. The intracellular concentration of oxidized glutathione in type II cells was also elevated. Acute lung injury was decreased by glutathione supplementation prior to endotoxin administration in this model. It was also determined that chronic ethanol ingestion altered surfactant synthesis and secretion, tissue viability and tissue remodeling during endotoxemia. Alcohol may also predispose to acute lung injury by altering alveolar-capillary permeability. Alveolar type II cells from ethanol-fed animals form a more permeable monolayer and have decreased alveolar fluid clearance and increased protein leak across the monolayer.

Table I Data presented are SOFA scores (numbers are in mean standard).

\begin{tabular}{lll}
\hline Category & Alcohol abuse & No alcohol abuse \\
\hline Coagulation & 2.34 & 1.72 \\
Renal & 1.7 & 1.66 \\
Liver & 1.64 & 1.17 \\
CNS & 2.91 & 2.38 \\
Cardiovascular & 2.27 & 2.22 \\
Total non-pulmonary & 9.42 & 8.05 \\
\hline
\end{tabular}

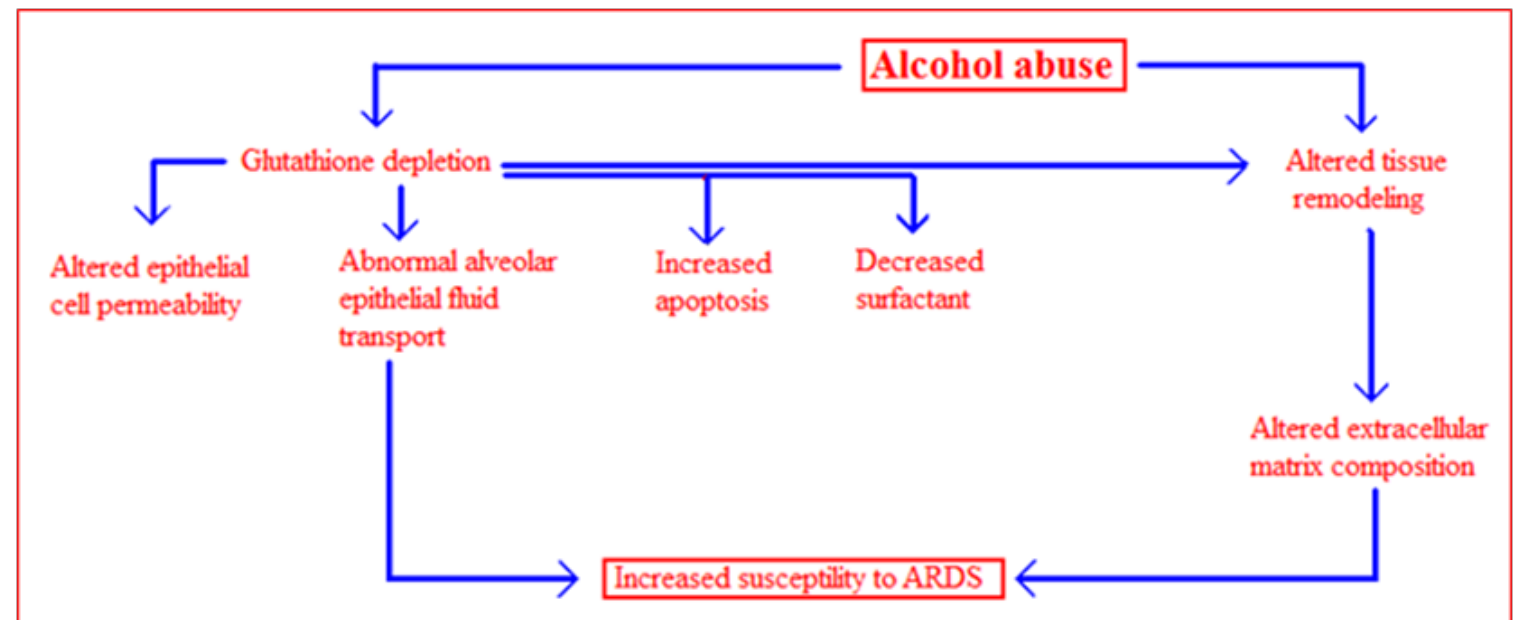

Figure 4 Possible mechanisms of alcohol-induced lung injury. The effects of chronic ethanol ingestion on the lung may be multifactorial. Alcohol abuse causes a decrease in glutathione levels in the lung, consequently alveolar type II epithelial cell functions. This leads to changes in epithelial cell permeability, decreased alveolar fluid clearance, decreased cell viability and decreased surfactant production. Chronic ethanol ingestion is also associated with the activation of tissue remodeling, resulting in alterations to extracellular matrix composition. All these alterations are thought to increase susceptibility to ARDS.

To evaluate whether the observations made in animals' model of alcohol ingestion carried over to human subjects, a series of studies were carried out. Subjects with a history of alcohol abuse and no medical history of cardiac, hepatic, renal or pulmonary disease, HIV positivity, diabetes mellitus or hypertension underwent bronchoalveolar lavage. The median total glutathione concentration in the epithelial lining fluid was lower in the patients with a history of alcohol abuse compared to smoking matched controls. In addition, the median percentage of oxidized glutathione was higher, indicating increased usage of glutathione in the epithelial lining fluid of the lung. However, the subjects with a history of alcohol abuse abstained from ethanol for 7 days and had repeat bronchoscopy it was found that the concentration of glutathione in the epithelial lining fluid remained diminished, indicating that pulmonary glutathione stores are not 
quickly restored during abstinence. In addition, the concentration of total protein in the epithelial lining fluid from individuals with a history of alcohol abuse was increased, suggesting an association between alcohol abuse and disruption of the alveolar-capillary barrier function.

Alcohol abuse also alters the pulmonary response to oxidative stress during acute lung injury. Endothelial selectin is unique among selectin adhesion molecules in that its expression is restricted to endothelial cells. ARDS patients with history of alcohol abuse have elevated serum endothelial-selectin levels in both plasma and bronchoalveolar lavage fluid when compared to non-alcoholic ARDS patients, indicating either increased endothelial activation or increased alveolar capillary permeability due to chronic exposure to alcohol. In addition, alcohol abuse is associated with an increased inflammatory response to injury during trauma. In studies of trauma patients, serum levels of IL-6, IL10 and serum endothelial-selectin were all increased in patients with a history of alcohol abuse on admission to the ICU when compared to non-alcoholics. ${ }^{4}$ Alcohol consumption and development of ALI/ ARDS: During hospital stay, ARDS developed in 13 out of $97(13 \%)$ patients with a significant alcohol consumption history, compared to only 64 out of $1,260(5 \%)$ nonalcoholic or patients consuming $<14$ drink/week. When stratified according to amount of alcoholic drinks per day alcohol consumption and the development of ARDS demonstrated a dose response relationship (Figure 5). This was held true when adjusted for other known ARDS risk factors (aspiration, chemotherapy, high-risk surgery, pancreatitis, sepsis, shock). Patients who reported drinking less than two drinks per day $(<14$ drinks per week), was had a similar incidence of ARDS compared to nondrinkers (5.6 \%). Anyhow, to further investigate the role of potential confounding variables, a multivariate logistic regression model was fit for the development of ARDS. After adjusting for differences in well-known underlying ARDS risk factors (aspiration, chemotherapy, high-risk surgery, pancreatitis, sepsis and shock), smoking, cirrhosis and gender, there is an effect of a positive history of significant alcohol consumption on the development of ARDS remained significant. ${ }^{5}$

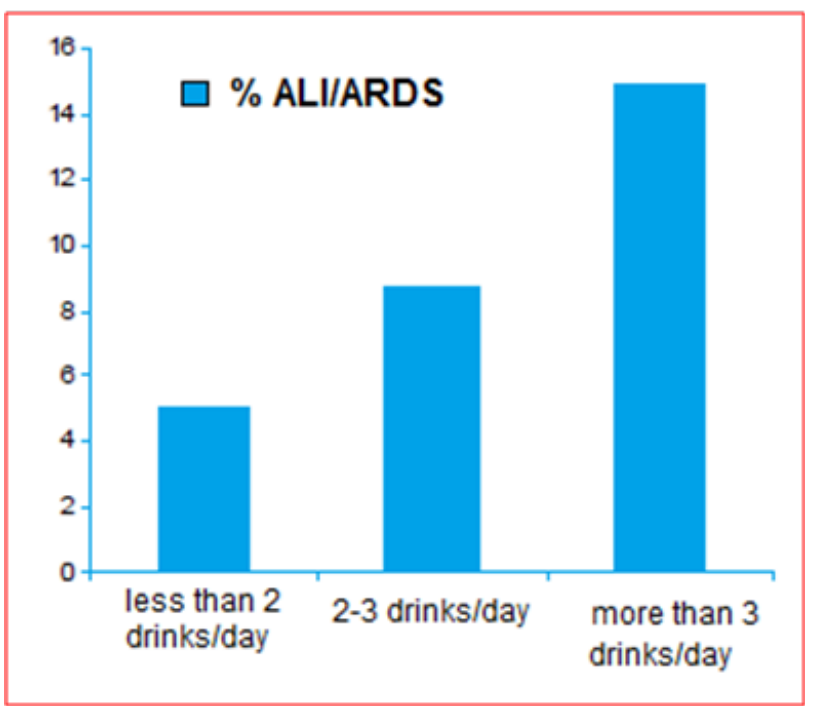

Figure 5 Dose response relationship of alcohol consumption on the development of ARDS.

Chronic alcoholism alters systemic and pulmonary glutathione redox status: The lungs are constantly exposed to environmental and endogenous oxidants. The ability to neutralize these oxidants is essential to maintain pulmonary health. There are major differences between cellular and extracellular compartments in concentration of the thiol/disulfide systems and the relative redox states. Since Cantin and colleagues' report, published in 1987, on the high glutathione concentration in the airspace (more than 20-fold than that in the plasma and over $90 \%$ in the reduced form), glutathione has been considered a primary antioxidant in the alveolar space. Although the alveolar space is exposed to the oxidants present in cigarette smoke, the alveolar glutathione pool is increased by $80 \%$ with no significant oxidant stress, as evidenced by the minimal shift toward the oxidized moiety oxidized glutathione (GSSG). In the airspace, this high glutathione concentration defends the lung against endogenously produced oxidants as well as environmental oxidants. Limited availability of glutathione in the alveolar space is associated with many pulmonary diseases, including cystic fibrosis, acute respiratory distress syndrome, chronic obstructive pulmonary disease, idiopathic pulmonary fibrosis, idiopathic interstitial pneumonia, and diffuse fibrosing alveolitis.

Previous studies reported about 3-fold elevation in the risk for ARDS in otherwise healthy alcohol abusers. Although the underlying cause remains unclear, recent research has elucidated several potential contributors, with one of the major causes being chronic oxidative stress. Nevertheless, the alcoholic lungs in ethanol-fed animal models are under increased oxidative stress, have altered nitric oxide metabolism, express abnormal amounts of transforming growth factor TGF- $\beta$, and are in a chronic proinflammatory state, with up-regulated expressions of IL- $1 \beta$ and tumor necrosis factor TNF- $\alpha$. In this animal model, chronic ethanol ingestion depleted the glutathione pool in the alveolar epithelial lining fluid by as much as $80 \%$. In clinical studies, chronic alcohol abuse resulted in a similar $80 \%$ decrease in the glutathione pool of bronchoalveolar lavage fluid in human. This decrease in glutathione was accompanied by a 4-fold increase in GSSG and was indicative of chronic oxidant stress. Altered homeostasis of the glutathione/GSSG thiol pair was present even after 1 week of abstinence and was independent of presence of cirrhosis. Therefore, these studies demonstrated that a history of alcohol abuse resulted in chronic oxidant stress in the alveolar space. Maccarrone and Ullrich suggested that the consideration of the redox state may be more reflective of the physiologically and pathological effects of oxidative stress than the mere absolute amounts of antioxidants and oxidants. ${ }^{6}$

In trauma patients, the risk of pulmonary complications, such as respiratory failure requiring mechanical ventilation, is higher if there is a history of alcohol abuse. In septic shock patients, $70 \%$ of alcoholdependent patients developed ARDS compared with 31\% among others. After controlling for potential confounding variables, the relative risk of ARDS associated with chronic alcohol abuse was 3.7. In these studies, a history of alcohol abuse alone did not result in acute lung injury. Rather, alcohol abuse increased the incidence and severity of ARDS when superimposed on a second injury, such as trauma, sepsis, or aspiration. It was found that depletion of plasma glutathione has previously been commonly reported. Concurrent alcohol abuse and smoking significantly increased the percentage of the glutathione pool present as GSSG. This increase in plasma \%GSSG was mirrored in smoking alcoholics by a similar shift in the redox potential of the glutathione/GSSG pair toward an oxidized state $(\sim 17 \mathrm{mV})$. Therefore, the significantly more oxidized redox potential of the glutathione/ GSSG pair and the greater \%GSSG suggested a systemic oxidative 
stress, particularly in alcohol abusers who also smoked, a behavior characteristic of $80 \%$ of all alcoholics (Figures $6 \mathrm{~A} \& 6 \mathrm{~B}$ ). As observed by other investigators, the glutathione concentration in the epithelial lining fluid was 140-fold greater than that present in the plasma, with less than $5 \%$ of the epithelial lining fluid pool present as the oxidized moiety. After correction for dilution by the lavage procedure, the redox potential of the epithelial lining fluid was approximately-190 $\mathrm{mV}$, a redox potential that approaches that of tissues ( -185 to -258 $\mathrm{mV})$. When the glutathione/GSSG redox potential of the plasma and the epithelial lining fluid were compared, the epithelial lining fluid was $30 \mathrm{mV}$ more reduced than plasma.

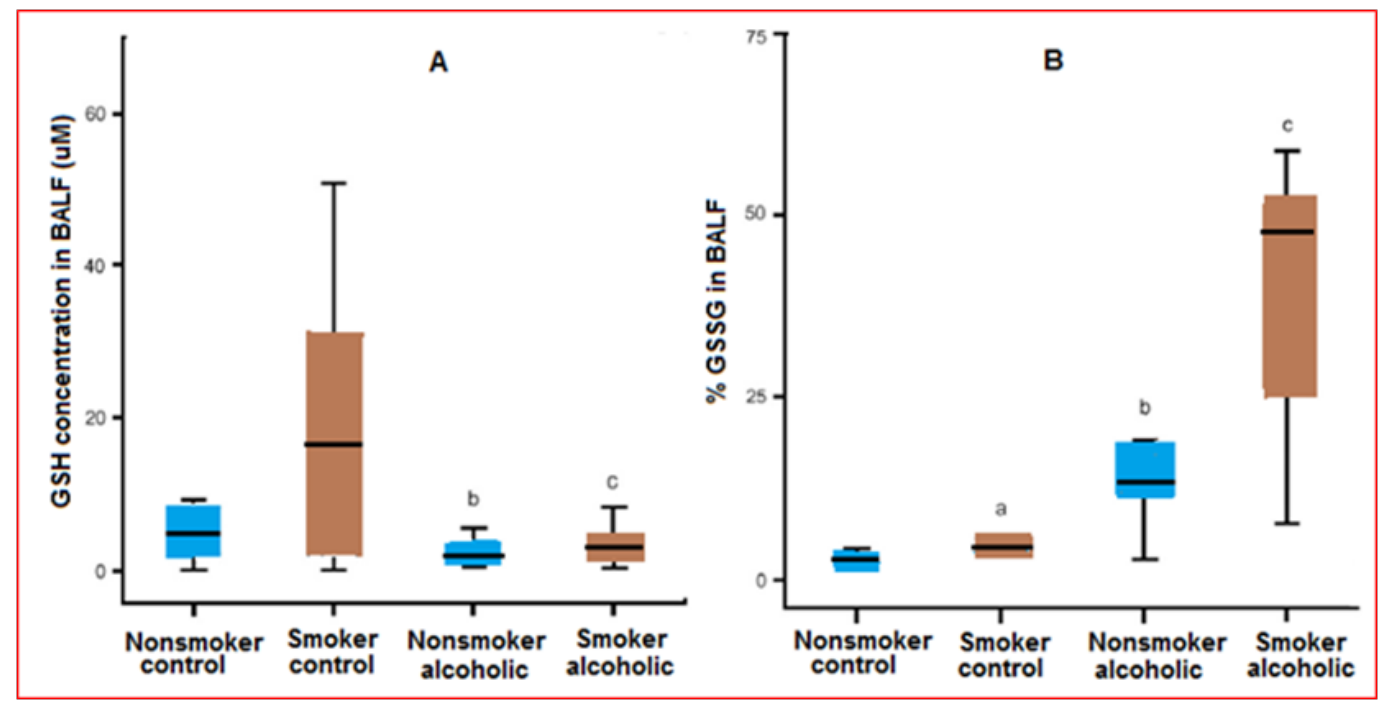

Figure 6 (A) Glutathione (GSH) concentrations in the bronchoalveolar lavage fluid (BALF). The GSH concentrations in the BALF of chronic alcoholics and control subjects with or without a smoking history were determined by HPLC. The data were presented as box plots with median lines, the lower and upper box edge for the first and third quartiles, respectively, and whiskers to indicate the smallest and largest values. (B) Percentage of oxidized glutathione (\%GSSG) in the bronchoalveolar lavage fluid (BALF). The percentage of the total GSH present as the oxidized moiety was determined in the BALF of chronic alcoholics and controls $(\% \mathrm{GSSG}=[\mathrm{GSSG}] /([\mathrm{GSH}]+[\mathrm{GSSG}]) \times 100)$. Data are presented as box plots.

In the bronchoalveolar lavage fluid, a smoking history is associated with a significant increase in the glutathione pool, with $98 \%$ remaining in the reduced form. However, this smoking-associated increase in glutathione was accompanied by an increase in GSSG. As a result, smoking was not associated with a significant shift in the glutathione/ GSSG redox potential in the bronchoalveolar lavage fluid or in the epithelial lining fluid of the control patients. In previous studies, a history of alcohol abuse was associated with an $80 \%$ decreased in the glutathione content of the lavage fluid and a fourfold increase in the \%GSSG. However, a similar decrease in the glutathione pool was observed in those subjects who abused alcohol. Furthermore, this decrease in glutathione associated with alcohol abuse was independent of their smoking history. When the GSSG pool was assessed, alcohol abuse resulted in a 3-4-folds increase in those subjects, independent of their smoking history. When the glutathione/GSSG redox potential of the bronchoalveolar lavage fluid or the epithelial lining fluid was determined, the alveolar lining fluid was approximately $40 \mathrm{mV}$ more oxidized in subjects with a history of alcoholism. This dramatic increase in alveolar oxidant stress was independent of their smoking histories. Therefore, the epithelial lining fluid redox potential of subjects who abused alcohol was only $15 \mathrm{mV}$ more reduced than the corresponding plasma sample. This was in sharp contrast to a 30$\mathrm{mV}$ epithelial lining fluid-to-plasma potential difference observed in control subjects.

Human studies undoubtedly require consideration of many factors that may contribute to the observed findings. In these subjects, there are many dietary factors that may alter plasma thiol state, including sulfur amino acid intake, availability of glutamine, dietary antioxidants, inducers of glutathione synthesis, and redox active micronutrients such as selenium, flavin, and niacin. Chronic alcoholism has been associated with deficiency in folate and other B vitamins that may participate in methionine and cysteine synthesis. Specific transporters move extracellular amino acids into the cells, and although studies in alcoholic micropig models have suggested down-regulation of transporters involved in folate and B-vitamin absorption, the effects of alcohol on these mechanisms in the lung remain unstudied. Evaluations of these numerous contributors in human studies would better explain the observations and reveal effective targets to decrease the risk of ARDS in this vulnerable population ${ }^{7}$

In other aspect, factors that predispose patients to ARDS include chronic alcohol abuse, a co-morbid variable known to increase the incidence of ARDS 2-4-fold in critically ill patients. While chronic alcohol ingestion likely increases ARDS incidence through multiple mechanisms, previous studies in animals' model of chronic alcohol ingestion, it was indicated that oxidative stress plays a key role in susceptibility to lung injury. In experimental animals, chronic alcohol ingestion enhanced sepsis-induced barrier dysfunction in the lung, a derangement attenuated by treatment with glutathione precursors. Chronic alcohol ingestion also altered the production of both reactive oxygen and nitrogen species in the lung through increased expression of NADPH oxidase and endothelial nitric oxide synthase (eNOS), respectively. These findings have led to the working hypothesis that chronic ethanol ingestion primes the lung by increasing the expression of enzymatic sources of reactive species. These alcoholinduced derangements while not sufficient to cause lung injury by themselves, contribute to susceptibility to injury caused by subsequent inflammatory stimuli. ${ }^{8}$ 
Zinc and alcohol-mediated alveolar macrophage dysfunction: There is a potential mediator of the macrophage dysfunction that is inherent to the alcoholic lung phenotype which is the zinc deficiency. Zinc is a key participant in normal host immune response, is critical for normal protein metabolism, is a co-factor required for the function of more than 300 metalloenzymes, and is necessary for membrane integrity. Additionally, adequate zinc levels are essential for healthy immune function, including both innate defenses (such as the alveolar macrophage) and adaptive defenses such as those provided by $\mathrm{T}$ and $\mathrm{B}$ lymphocytes. Alcohol abuse is often complicated by malnutrition, and zinc deficiency has been postulated to cause some of the skin changes and immunodeficiency associated with alcoholic liver disease. The primary source of zinc in the diet is meat, although vegetarians eating a balanced diet can have adequate intake and zinc transport in the intestine can be up-regulated significantly when dietary zinc is low. Therefore, the assumption has been that the zinc deficiency in alcoholics is the result of poor nutrition. However, there is recent experimental evidence which revealed that chronic alcohol ingestion significantly decreases the expression of the primary zinc transporter in the intestinal epithelium, and that systemic zinc deficiency develops even when adequate zinc is present in the diet. Nevertheless, it is suggested that individuals who abuse alcohol could develop zinc deficiency even with a balanced diet, and that this zinc deficiency would be even more profound if their diet is poor. Consistent with this are other experimental findings in cell culture, animal models, and humans that alcohol can interfere with zinc transport and/or bioavailability, including maternal transfer to the fetus across the placental barrier.

It is recently determined that chronic alcohol ingestion in experimental animals alters the expression of zinc transporters and causes significant intracellular zinc deficiency in the alveolar macrophage. Remarkably, dietary zinc supplementation in these animals (in doses that are comparable to those used to treat zinc deficiency clinically) restored macrophage zinc levels and bacterial phagocytic function. In a subsequent study with even stronger potential clinical implications, dietary zinc supplementation restored the ability of these alcohol-fed animals to clear a bacterial challenge from their lungs as efficiently as animals on a control diet. In that study, dietary zinc supplementation also improved nuclear binding of PU.1, the master transcription factor for GM-CSF signaling, and of $\mathrm{Nrf}$, the master transcription factor required to activate the antioxidant response element.

Taken together, the experimental findings discussed above reveal a complex interplay between alcohol-induced zinc deficiency and two fundamental signaling pathways that are required for strong host immune capacity within the alveolar macrophages. Specifically, it appears that alcohol interferes with dietary zinc absorption in the gut but also impairs zinc transport into the alveolar space and its uptake by the alveolar macrophage (Figure 7). As consequence of this intracellular zinc deficiency, GM-CSF signaling is dampened as reflected by decreased surface expression of its receptor as well as decreased expression and binding of its master transcription factor, PU.1. As GM-CSF signaling is critical to prime the mature immune function of the alveolar macrophage, the result is decreased phagocytic function. In parallel, alcohol-induced zinc deficiency dampens activation of the anti-oxidant response element by decreasing the expression and nuclear binding of its master transcription factor, Nrf2.
As a result, the production of a broad array of critical antioxidants, including glutathione, is severely limited and leaves the macrophage susceptible to the oxidative stress that accompanies acute insults. ${ }^{9}$

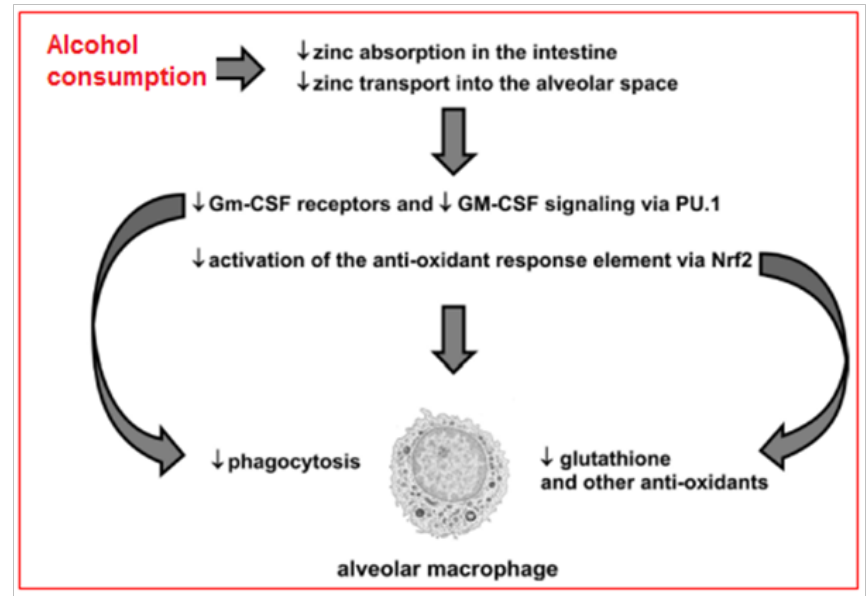

Figure 7 Hypothetical schemes for alcohol-mediated alveolar macrophage dysfunction. There is evolving experimental evidence that chronic alcohol abuse inhibits transport-mediated absorption of dietary zinc in the small intestine and, in parallel, decreases zinc transport into the alveolar space and uptake by the alveolar macrophage. As consequence, the surface expression of GM-CSF receptors and the intracellular signaling of GM-CSF, via its master transcription factor, PU.I, are both decreased and thereby impair alveolar macrophage phagocytic capacity. In parallel, the alcohol-mediated zinc deficiency inhibits activation of the anti-oxidant response element by decreasing signaling through its master transcription factor, Nrf2. As a result, the alveolar macrophage becomes profoundly deficient in glutathione and other anti-oxidants, and is therefore vulnerable to further cellular damage and dysfunction during the acute oxidative stress imposed by pneumonia or sepsis.

\section{Acknowledgments}

None.

\section{Conflicts of interest}

Author declares that there is no conflicts of interest.

\section{References}

1. Moss M, Bucher B, Moore FA, et al. The role of chronic alcohol abuse in the development of acute respiratory distress syndrome in adults. JAMA. 1996;275(1):50-54.

2. Burnham EL, Kovacs EJ, Davis CS. Pulmonary cytokine composition differs in the setting of alcohol use disorders and cigarette smoking. $A m$ J Physiol Lung Cell Mol Physiol. 2013;304(12):L873-L882.

3. Afshar M, Smith GS, Terrin ML, et al. Blood Alcohol Content, Injury Severity and Acute Respiratory Distress Syndrome. J Trauma Acute Care Surg. 2014;76(6):1447-1455.

4. Esper A, Burnham EL, Moss M. The effect of alcohol abuse on ARDS and multiple organ dysfunctions. Minerva Anesthesiol. 2006;72(6):375-381.

5. Thakur L, Kojicic M, Thakur SJ, et al. Alcohol consumption and development of acute respiratory distress syndrome: a population-based study. Int J Environ Res Public Health. 2009;6(9):2426-2435.

6. Maccarrone M, Ullrich V. Redox regulation in disease and ageing. Cell Death Differ. 2004;11(8):949-951. 
7. Yeh MY, Burnham EL, Moss M, et al. Chronic alcoholism alters systemic and pulmonary glutathione Redox status. Am J Respir Crit Care Med. 2007;176(3):270-276.

8. Wagner MC, Yeligar SM, Brown LA, et al. PPAR $\gamma$ ligands regulate NADPH oxidase, eNOS, and Barrier function in the lung following chronic alcohol ingestion. Alcohol Clin Exp Res. 2012;36(2):197-206.
9. Mehta AJ, Guidot DM. Pathophysiology review series: alcohol abuse, the alveolar macrophage, and pneumonia. Am J Med Sci. 2012;343(3):244-247. 